\title{
Dengue Prediction Using Deep Learning With Long
}

\section{Short-Term Memory}

\author{
Abdulrazak Yahya Saleh \\ FSKPM Faculty \\ Universiti Malaysia Sarawak (UNIMAS) \\ Kota Samarahan, Sarawak, Malaysia \\ ysahabdulrazak@unimas.my
}

\author{
Lim Baiwei \\ FSKPM Faculty \\ Universiti Malaysia Sarawak (UNIMAS) \\ Kota Samarahan, Sarawak, Malaysia \\ lim.omarz@gmail.com
}

\begin{abstract}
Dengue is a severe infectious disease on the rise in Malaysia, and there is a demand for artificial intelligence to support the health system. However, the application of deep learning, specifically Long Short-Term Memory (LSTM) time series forecasting, has not been explored by many in dengue prediction studies. However, considering the availability of daily weather data being collected, the ability of LSTM to capture long term dependencies can be leveraged in forecasting dengue cases. Therefore, this study investigates the performance and viability of LSTM time series forecasting on predicting dengue cases. An LSTM model is developed and evaluated to be compared to a Support Vector Regression (SVR) model by utilising the availability of a dengue dataset consisting of weather and climate data. The results indicated LSTM time series forecasting performed better than SVR, with R2 and MAE scoring 0.75 and 8.76. In short, LSTM has shown better performance and, in addition, capturing trends in the rise and fall of dengue cases. Altogether, this research could contribute to the fight against the increase of dengue cases without relying on forecasted weather data but instead, history.
\end{abstract}

Keywords - LSTM, Time series forecasting, Deep learning, dengue.

\section{INTRODUCTION}

Dengue is a severe disease that has plague Malaysia for many years as a tropical country. With the rise in popularity of machine learning, it is becoming an essential subject in researches to find ways of implementing it to aid the fight against dengue. There have been several types of research that have delved into the topic of dengue outbreak prediction. Still, most have focused on traditional machine learning techniques, and little to none have explored the possibilities of deep learning, another branch of machine learning. The deep learning technique to be studied in this research is implementing Long Short-Term Memory in time series forecasting.

According to WHO [1], "Dengue is a fast emerging pandemic-prone viral disease in many parts of the world.". It is a mosquito-borne viral infection that causes flu-like symptoms and can cause severe dengue, resulting in a fatality. The primary vector of dengue is the Aedes aegypti mosquito, transmitting the disease through the bites of an infected female. One of the most significant driving forces behind the spread of dengue is the weather. Malaysia is a tropical country with a warm ambient temperature and has been very satisfactory for the Aedes mosquito to breed and feed [2]. However, Malaysia's long history of battles against dengue has produced little results as the dengue fever pandemic in Malaysia has reached an all-time high [3]. In response to this, the Malaysian health minister expressed the need for artificial intelligence in dengue outbreak prediction to complement the existing healthcare system [4]. More Dengue Statistics can be found in figure 1 .

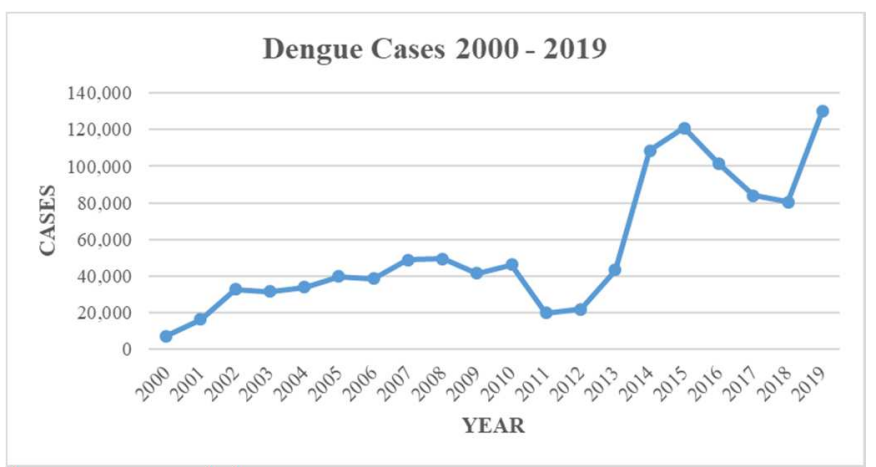

Fig 1. Dengue Statistics [5]

While there is a current application of artificial intelligence in outbreak prediction by a private company in Penang, it only achieves an accuracy of $80 \%$ using Bayesian networks [6]. Other than this, previous researches, recent and past in Malaysia, has only focused on the use of traditional machine learning methods such as decision tree [7], neural network models[8-10], regression [11], and adverse selection algorithm [12]. However, today, with the availability of big data and the latest advancement in deep learning, it has shown favourable results in predicting future outcomes and extracting highly nonlinear relationships [13]. Furthermore, deep understanding has also led to higher accuracy than non-deep learning approaches [14].

Therefore, this study proposes the use of deep learning, specifically Long Short-Term Memory (LSTM) time-series forecasting. With the availability of daily weather data collected sequentially, the advantage of LSTM in learning long term dependencies can be leveraged [15] to capture the trends in dengue outbreaks instead of relying on features alone to forecast. Another advantage of deep learning is achieving results without 